\title{
Vástagos estriados cónicos modulares en revisión de artroplastia total de cadera. Estudio multicéntrico. Resultados y complicaciones a mediano plazo
}

\author{
Santiago L. Iglesias, " Fernando Vanoli, " Leonel Pérez Alamino,," Ignacio Pioli," José Gómez," Fernando A. Lopreite," \\ Pablo Maletti," Hernán del Sel,"* Bartolomé L. Allende* \\ "Servicio de Ortopedia y Traumatología, Sanatorio Allende, Córdoba, Argentina \\ ${ }^{*}$ Servicio de Ortopedia y Traumatología, Hospital Británico de Buenos Aires, Ciudad Autónoma de Buenos Aires, Argentina \\ "Traumatología del Comahue, Neuquén, Argentina
}

\section{RESUMEN}

Introducción: Debido al crecimiento exponencial del número de artroplastias de cadera, se espera una mayor cantidad de revisiones en las próximas décadas. Los vástagos cónicos estriados modulares se han vuelto populares en la última década por sus resultados favorables. El objetivo de este estudio es evaluar los resultados, las complicaciones y la tasa de supervivencia de estos vástagos en las revisiones de cadera, con un seguimiento a mediano plazo. Materiales y Métodos: Estudio multicéntrico, retrospectivo. Se incluyeron 182 pacientes a quienes se les realizó una cirugía de revisión de cadera entre 2007 y 2017 . Se colocaron 185 vástagos cónicos estriados de fijación distal. El déficit de stock óseo femoral se clasificó según Paprosky y Burnett; y las facturas periprotésicas, según la clasificación de Vancouver. Se evaluó a los pacientes clínicamente con el Harris Hip Score (HHS) y con radiografías a los 3 meses y anualmente para evaluar la estabilidad del vástago, la subsidencia y el aflojamiento, así como la consolidación de la osteotomía. Resultados: Seguimiento medio 55.18 meses. EI HHS posoperatorio tuvo una media de 80,28 (DE = 12,8, IC95\% 78,5-82,97). No hubo complicaciones posoperatorias en el 75,4\% de los pacientes. Las complicaciones más frecuentes fueron inestabilidad (7,6\%) y subsidencia del implante $(11,5 \%)$. Al final del seguimiento, el $95,05 \%$ de los pacientes tenía un implante estable. Conclusiones: Los vástagos cónicos estriados modulares de fijación distal proporcionan una solución confiable, reproducible y duradera para el manejo de revisiones de componentes femorales a medio plazo.

Palabras clave: Articulación de cadera; artroplastia; reemplazo articular; fémur; falla de prótesis.

Nivel de Evidencia: IV

\section{Modular Fluted Tapered Stems in Revision Total Hip Arthroplasty. Multicenter Study.}

Medium-term Results and Complication Rate

\section{ABSTRACT}

Background: The number of arthroplasties performed every year is increasing, therefore a greater number of revisions is expected in the coming decades. Modular fluted tapered stems have become the gold standard for their results in different series of patients. The objective of this article is to evaluate the results, complications and the survival rate of these stems in hip revisions with a medium-term follow-up. Materials and Methods: Retrospective, multicenter analysis. One hundred eighty-two patients who had undergone hip revision surgery between 2007 and 2017 were included. One hundred eighty-five modular fluted tapered stems were placed. Femoral bone stock defects were classified according to Paprosky and Burnett; and periprosthetic femur fractures according to Vancouver classification. Patients were evaluated clinically with Harris Hip Score (HHS) and radiographically 3 months after surgery and every year to assess stem stability, subsidence and loosening, as well as osteotomy healing. Results: Average follow-up was 55.18 months. Postoperative HHS had an average of 80.28 (SD $=12.8,95 \% \mathrm{Cl}=[78.5,82.97])$. There were no postoperative complications in $75.4 \%$ of the patients. The most frequent complications were instability in $7.6 \%$ and implant subsidence in $11.5 \%$. At the end of the follow-up, $95,05 \%$ of the patients had a stable implant. Conclusion: Modular fluted tapered stems provide a reliable, reproducible solution for the management of femoral component revisions at medium-term.

Key words: Hip joint; arthroplasty; hip replacement; femur; prosthesis failure.

Level of Evidence: IV

Recibido el 15-5-2020. Aceptado luego de la evaluación el 14-9-2020 • Dr. SANTIAGO L. IGLESIAS • santiglesias07@ hotmail.com

https://orcid.org/0000-0001-6698-2914

Cómo citar este artículo: Iglesias SL, Vanoli F, Pérez Alamino L, Pioli I, Gómez J, Lopreite FA, Maletti P, del Sel H, Allende BL. Vástagos estriados cónicos modulares en revisión de artroplastia total de cadera. Estudio multicéntrico. Resultados y complicaciones a mediano plazo. Rev Asoc Argent Ortop Traumatol 2021;86(2):197-206.

https://doi.org/10.15417/issn. 1852-7434.2021.86.2.1118 


\section{INTRODUCCIÓN}

La artroplastia total de cadera es un procedimiento altamente eficaz, alivia el dolor, mejora la función y la calidad de vida, y le otorga al paciente una razonable satisfacción e independencia. A pesar del éxito de la artroplastia total de cadera, un pequeño porcentaje de pacientes requiere múltiples cirugías, a menudo, relacionadas con el fracaso del componente femoral. Debido al crecimiento exponencial del número de artroplastias de cadera, se espera una cantidad mayor de revisiones en las próximas décadas. ${ }^{1}$ Si bien ya se ha descrito la revisión del componente femoral con el uso de implantes cementados, ${ }^{2}$ en la actualidad, las prótesis no cementadas han ganado gran popularidad, y logran mejores resultados clínicos y radiológicos. ${ }^{3-5}$

Existen numerosas técnicas quirúrgicas a la hora de pensar en la revisión del componente femoral, como vástagos cementados con injerto óseo o sin él, ${ }^{6-10}$ aloinjerto-prótesis ${ }^{11,12}$ y vástagos no cementados de diferentes diseños, por ejemplo, vástagos porosos extensamente recubiertos ${ }^{13-15}$ o vástagos cónicos estriados modulares y no modulares. ${ }^{16-21}$

Los vástagos cónicos estriados modulares se han vuelto populares en la última década por sus resultados favorables en pequeñas series ${ }^{17,20,22}$ y la reproducibilidad de su técnica. ${ }^{23}$ Se ha informado que tienen tasas más bajas de stress-shielding y subsidencia del implante.

El objetivo de este estudio fue evaluar los resultados, las complicaciones y la tasa de reintervención con el uso de vástagos cónicos estriados de fijación distal en las revisiones del componente femoral llevadas a cabo en tres centros de referencia y Nivel I, en nuestro país, luego de un seguimiento a mediano plazo.

\section{MATERIALES Y MÉTODOS}

Estudio multicéntrico, retrospectivo. Los criterios de inclusión fueron: pacientes sometidos a cirugía de revisión de cadera entre 2007 y 2017 a los cuales se les colocaron vástagos cónicos estriados de fijación distal, independientemente de la causa de la revisión, el sexo y las comorbilidades. Se incluyeron 182 pacientes a quienes se les colocaron 185 vástagos cónicos estriados de fijación distal. Todos fueron operados en tres centros de Nivel I por cirujanos especialistas en el área.

La planificación preoperatoria incluyó la evaluación radiográfica del implante previo y del déficit de stock óseo femoral que fue clasificado, según Paprosky y Burnett. ${ }^{24}$ De acuerdo con la técnica quirúrgica, se consideró necesaria una fijación diafisaria mínima de $5 \mathrm{~cm}$ para asegurar una correcta fijación distal; cuando esto no era posible, se decidió emplear otra técnica quirúrgica. ${ }^{25}$ Dos pacientes tenían un defecto tipo I de Paprosky; 40 casos, tipo II; 62, tipo IIIA; 34, tipo IIIB y 11, tipo IV.

Para las fracturas periprotésicas de fémur, utilizamos la clasificación de Vancouver. ${ }^{26}$ Un paciente tenía una fractura tipo B1; 16, tipo B2 y 19, tipo B3.

La subsidencia del implante se midió desde puntos de referencia fijos en la prótesis hasta puntos de referencia fijos en el fémur. ${ }^{27} \mathrm{El}$ aflojamiento del implante se definió como un hundimiento progresivo del implante o una línea radiolúcida continua alrededor del componente femoral. ${ }^{28}$

Todos los pacientes fueron operados en decúbito lateral, con abordaje posterolateral. Se utilizaron tallos modulares ZMR ${ }^{\circledR}$ (Zimmer $\left.{ }^{\circledR}\right)$, Restoration ${ }^{\circledR}\left(\right.$ Stryker $\left.{ }^{\circledR}\right)$, Reclaim ${ }^{\circledR}\left(\right.$ DePuy Synthes, Inc.), Prevision ${ }^{\circledR}$ (Aesculap $\left.{ }^{\circledR}\right)$, MPTM (Link $®), \operatorname{Arcos} \circledR(B i o m e t)$, según la preferencia de los cirujanos, al margen de la causa de la revisión femoral.

En el período posoperatorio inmediato, se permitió, de forma rutinaria, la descarga de peso completa, según la tolerancia, con un caminador independientemente del tipo de defecto óseo. A los pacientes con fracturas intraoperatorias se les indicó una descarga de peso del 50\% sobre la pierna afectada. Por otro lado, todos siguieron un estricto protocolo con precauciones de la movilidad de la cadera (flexión, abducción y rotación interna) por seis semanas. Los pacientes recibieron profilaxis antibiótica en dosis prequirúrgica y posquirúrgica inmediata, y profilaxis antitrombótica por 28 días.

El seguimiento clínico se realizó a las 3 y 6 semanas, 3 meses, 1 año y posteriormente controles anuales. Para la evaluación clínica se empleó el Harris Hip Score (HHS) ${ }^{29}$ y se tomaron radiografías a los 3 meses y anualmente para evaluar la estabilidad del vástago, la subsidencia y el aflojamiento, así como la consolidación de la osteotomía. 


\section{Análisis estadístico}

Se realizó un análisis exploratorio (medidas resumen, tablas de distribución de frecuencias y gráficos - diagramas de cajas [box plots], diagrama de barras e histograma) de la muestra de pacientes, caracterizando por sexo, edad, y las características indagadas.

Para estudiar la asociación entre el tipo de implante, su resultado luego del seguimiento, la presencia o no de intervención previa (y la cantidad), las causas de revisión femoral, la presencia de complicaciones, así como el sexo y la edad, se estimaron modelos logarítmicos lineales de Poisson (más de dos dimensiones o cruces de variables) y tablas de contingencia, con el test ji al cuadrado (asociación bidimensional), estableciendo un nivel de significación igual a 0,05 . Asimismo, se estimaron los coeficientes de correlación de Pearson cuando las variables fueron cuantitativas (HHS, tiempo de seguimiento, edad) y se testeó la diferencia de medias de estas según el sexo, el implante previo, etc., usando el test de Student y análisis de la varianza. Cuando la distribución fue normal, se obtuvieron, además, los intervalos de confianza usando un nivel de confianza del $95 \%$.

\section{RESULTADOS}

El seguimiento medio fue de 55.18 meses, valor que estuvo condicionado por un $10 \%$ de los pacientes que solo tuvieron una evaluación radiográfica de seguimiento antes del año posoperatorio, pero que, sin embargo, fueron controlados clínicamente por vía telefónica. La edad promedio era de 64.97 años. Ciento un pacientes (55,49\%) eran mujeres y 81 (44,51\%), hombres (Tabla). El 37\% de los 182 pacientes incluidos tenía como implantes previos vástagos no cementados, sin diferencias estadísticamente significativas en relación con el sexo (p=0,610). El 62\% y el $25 \%$ ya habían sido sometidos a cirugía en una o dos oportunidades, respectivamente, un $10 \%$ tenía tres o más cirugías previas. No hubo diferencias estadísticamente significativas en cuanto al sexo $(p=0,37)$ (Figura 1).

En relación con las causas de revisión femoral, la edad no fue un factor predisponente a este evento $(\mathrm{p}=0,745)$. Las cirugías de revisión del componente femoral fueron secundarias a aflojamiento aséptico ( 85 casos; 45,95\%), aflojamiento séptico (revisión en dos etapas) (54 casos; 29,19\%), fractura periprotésica (36 casos; 19,46\%), fracturas del vástago ( 7 casos; $3,78 \%$ ) e inestabilidad ( 3 casos; $1,62 \%)$. Cuando se correlacionó el sexo con la causa de la revisión, se observaron diferencias estadísticamente significativas $(\mathrm{p}=0,047)$ (Figura 2).

Tabla. Datos demográficos

\begin{tabular}{l|l}
\hline $\begin{array}{l}\text { Sexo } \\
\text { Femenino } \\
\text { Masculino }\end{array}$ & $\begin{array}{l}101(55,49 \%) \\
81(44,51 \%)\end{array}$ \\
\hline Edad media & 64,97 años \\
\hline $\begin{array}{l}\text { Defecto óseo femoral } \\
\text { Clasificación de Paprosky }\end{array}$ & \\
\hline $\begin{array}{l}\text { Tipo I } \\
\text { Tipo II }\end{array}$ & $2(1,34 \%)$ \\
\hline Tipo IIIA & $40(26,85 \%)$ \\
\hline $\begin{array}{l}\text { Tipo IIIB } \\
\text { Tipo IV }\end{array}$ & $62(41,61 \%)$ \\
\hline $\begin{array}{l}\text { Fractura periprotésica de fémur } \\
\text { Clasificación de Vancouver }\end{array}$ & $34(22,82 \%)$ \\
\hline Tipo B1 & $11(7,38 \%)$ \\
\hline Tipo B2 & \\
\hline Tipo B3 & \\
\hline Seguimiento medio & $1(2,78 \%)$ \\
\hline & $16(44,44 \%)$ \\
\hline & $19(52,78 \%)$ \\
\hline
\end{tabular}






Figura 1. Distribución de frecuencia de los pacientes según la cantidad de cirugías previas y el sexo (izquierda) y resultado del implante (derecha).

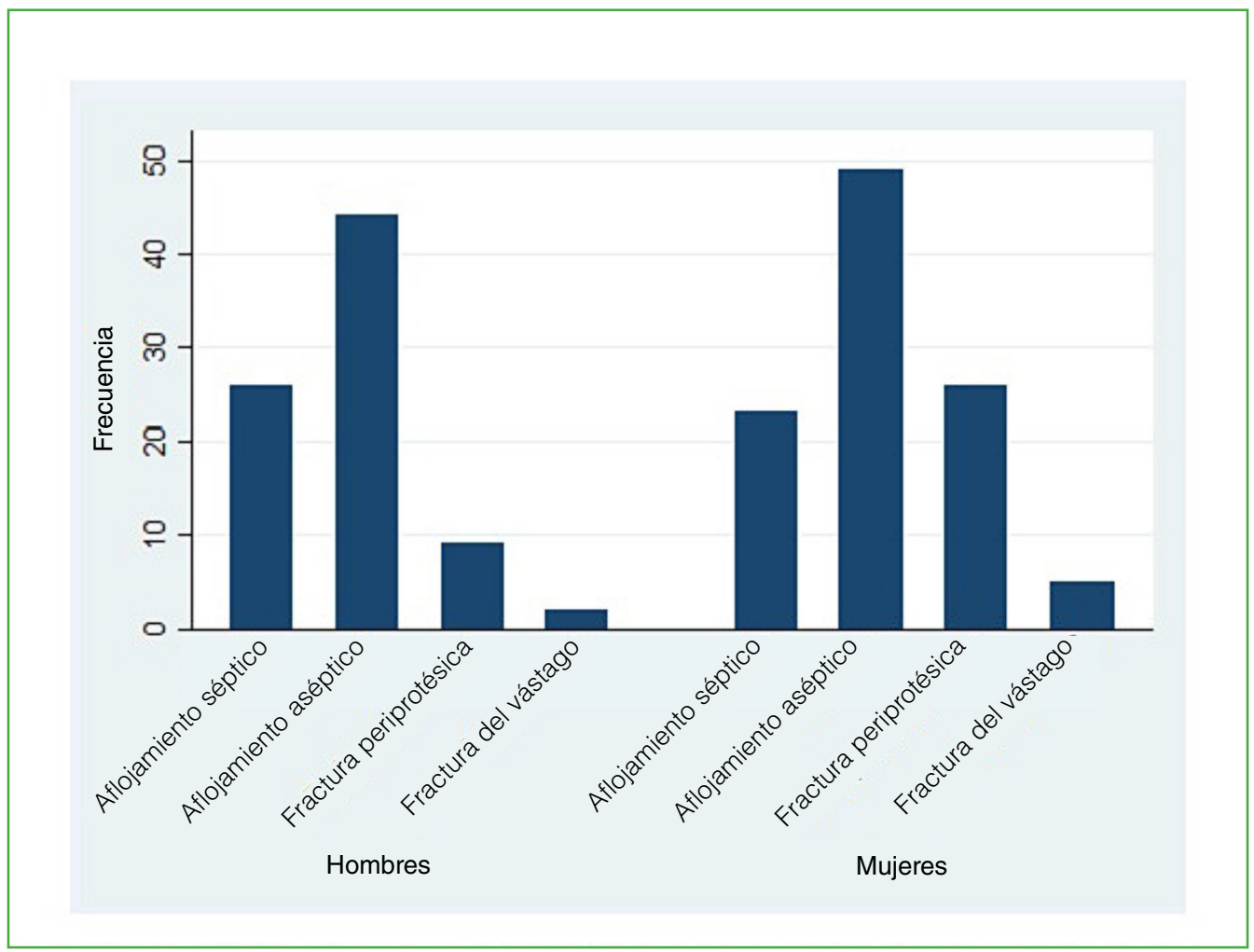

Figura 2. Distribución de frecuencia de los pacientes según las causas de revisión y el sexo. 
El HHS posoperatorio tuvo una media de 80,28 (desviación estándar $=12,8$; intervalo de confianza del 95\% [IC95\%] 78,5-82,97), un $25 \%$ de los pacientes estaba por debajo de 75,5 y otro $25 \%$ tenía valores superiores a $88,3$. No se encontraron diferencias estadísticamente significativas en relación con la edad ( $p=0,334)$, el tiempo de seguimiento (Figura 3), el implante previo $(\mathrm{p}=0,843)$ o la cantidad de cirugías previas ( $\mathrm{p}=0,33)$. El HHS tampoco se correlacionó con el sexo ( $\mathrm{p}=0$,394) (IC95\% 78,2-84,14 y 77,4-81,8 para hombres y mujeres, respectivamente).

$\mathrm{Al}$ analizar las complicaciones intraoperatorias, el 2,19\% (4 pacientes) tenía una fractura intraoperatoria en el momento de colocar el vástago, sin verse afectada la estabilidad definitiva del implante.

El 75,4\% de los pacientes no sufrió complicaciones posoperatorias. Catorce $(7,6 \%)$ tuvieron un episodio de inestabilidad. Esto influyó en el HHS posoperatorio que mostró diferencias significativas $(\mathrm{p}=0,047)$ con aquellos que no tuvieron luxaciones. Otra complicación observada fue la subsidencia/hundimiento del vástago (11,5\%), con un promedio de $4,1 \mathrm{~mm}$ al final del seguimiento.

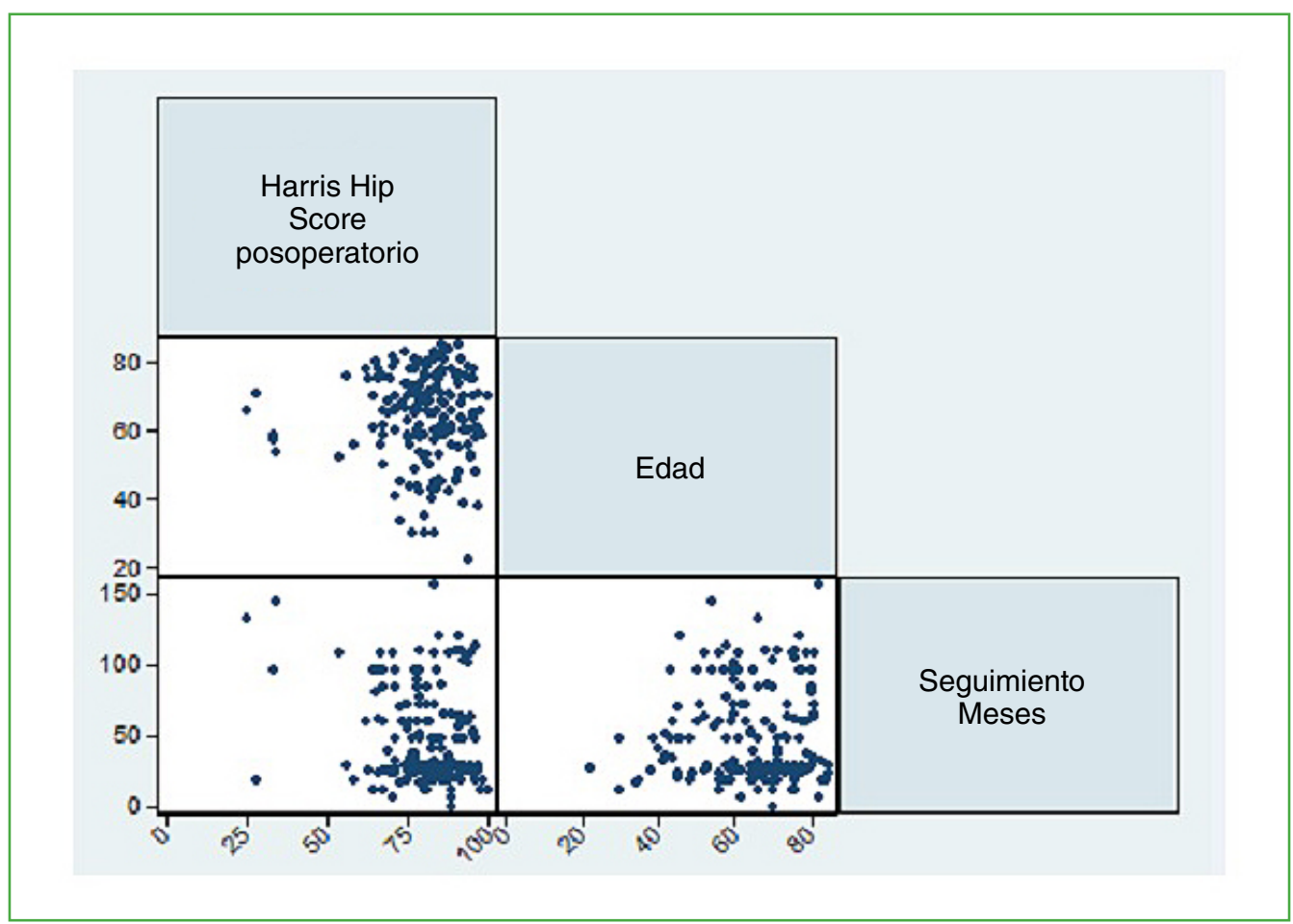

Figura 3. Diagrama de dispersión entre el Harris Hip Score posoperatorio, la edad y el tiempo de seguimiento (meses) de los pacientes.

Estas complicaciones no se asociaron con el sexo $(\mathrm{p}=0,882)$, la edad $(\mathrm{p}=0,372)$ o el implante previo $(\mathrm{p}=0,225)$ (Figura 4).

El déficit de stock óseo no mostró diferencias estadísticamente significativas en relación con el HHS posoperatorio $(\mathrm{p}=0,086)$. Por el contrario, los pacientes con fracturas periprotésicas tenían una tendencia hacia los valores más bajos del HHS (Figura 5).

Solo 14 pacientes requirieron una reintervención quirúrgica: cinco debido a inestabilidad donde no fue necesario revisar el vástago; cuatro como consecuencia de una infección profunda y cinco por subsidencia del implante y aflojamiento aséptico (a los 5, 6, 7, 9 y 15 meses, respectivamente).

Al final del seguimiento, el $95,05 \%$ de los pacientes incluidos en esta serie tenía implantes estables sin que fuera necesaria una nueva revisión (Figuras 6 y 7). 


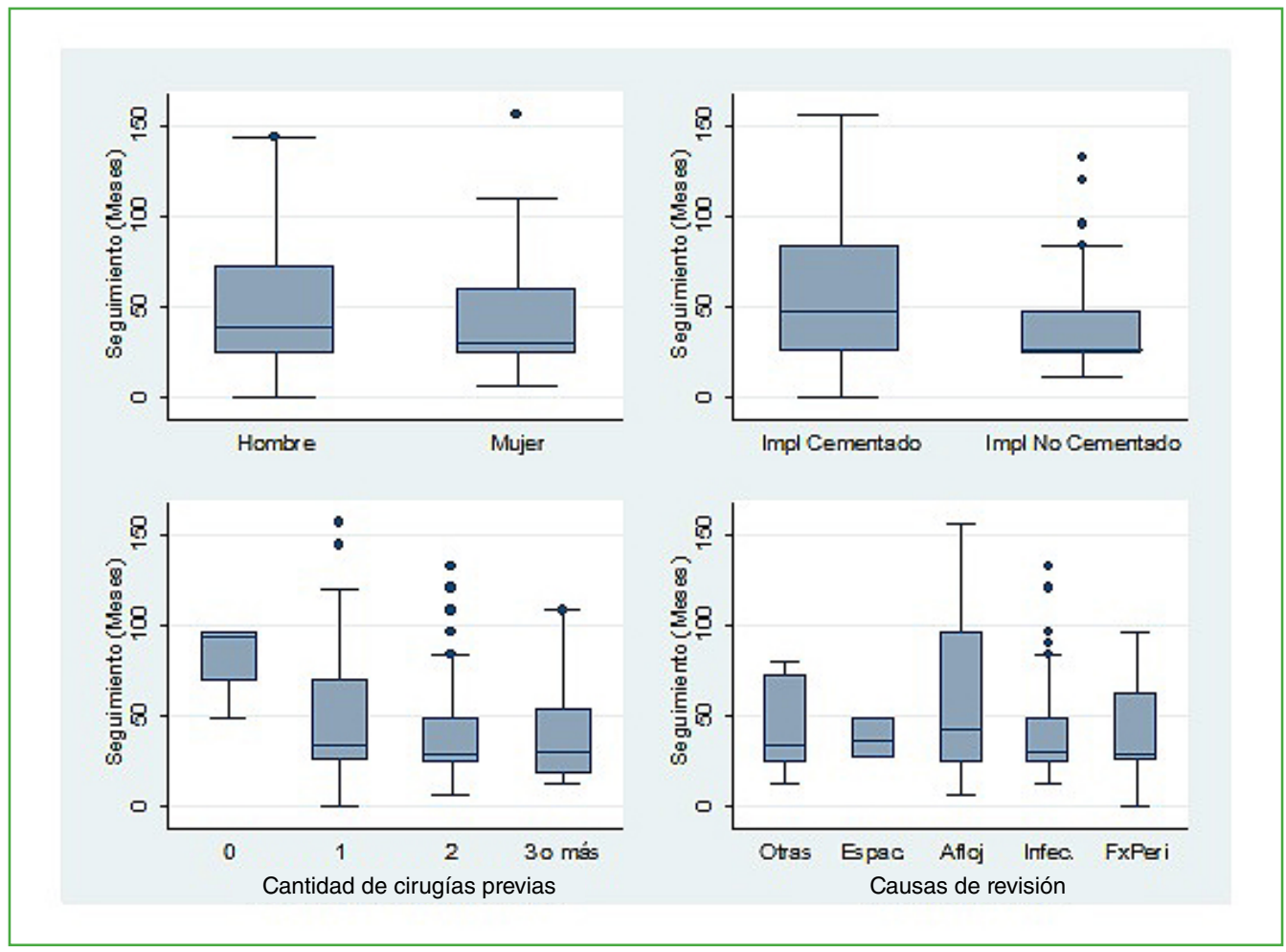

Figura 4. Diagrama de cajas del tiempo de seguimiento (meses) en función del sexo, el éxito del implante, la cantidad de cirugías previas y las causas de revisión de los pacientes.

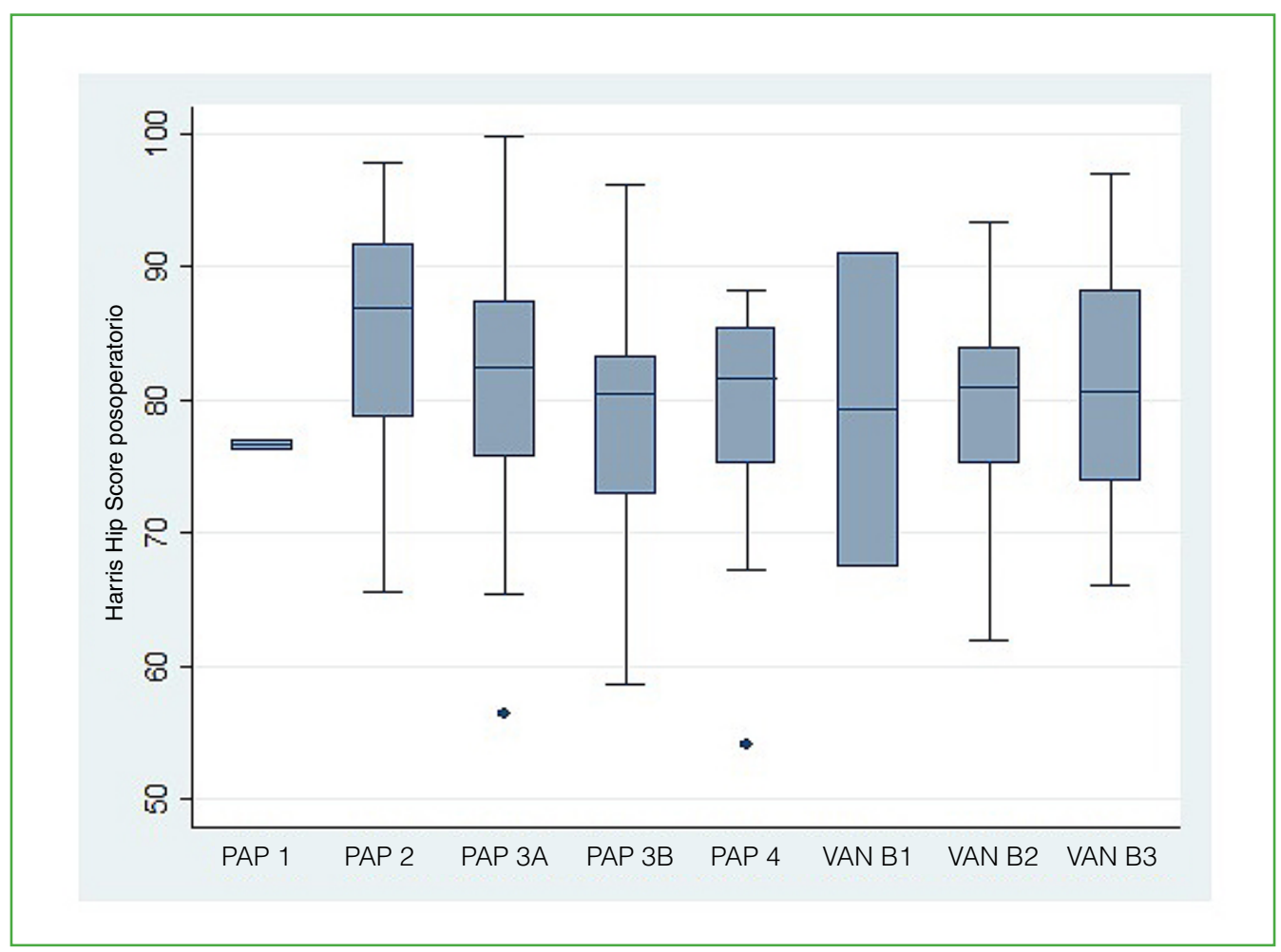

Figura 5. Diagrama de cajas del Harris Hip Score posoperatorio en función de la clasificación del implante colocado. $\mathrm{PAP}=$ Paprosky, VAN $=$ Vancouver. 


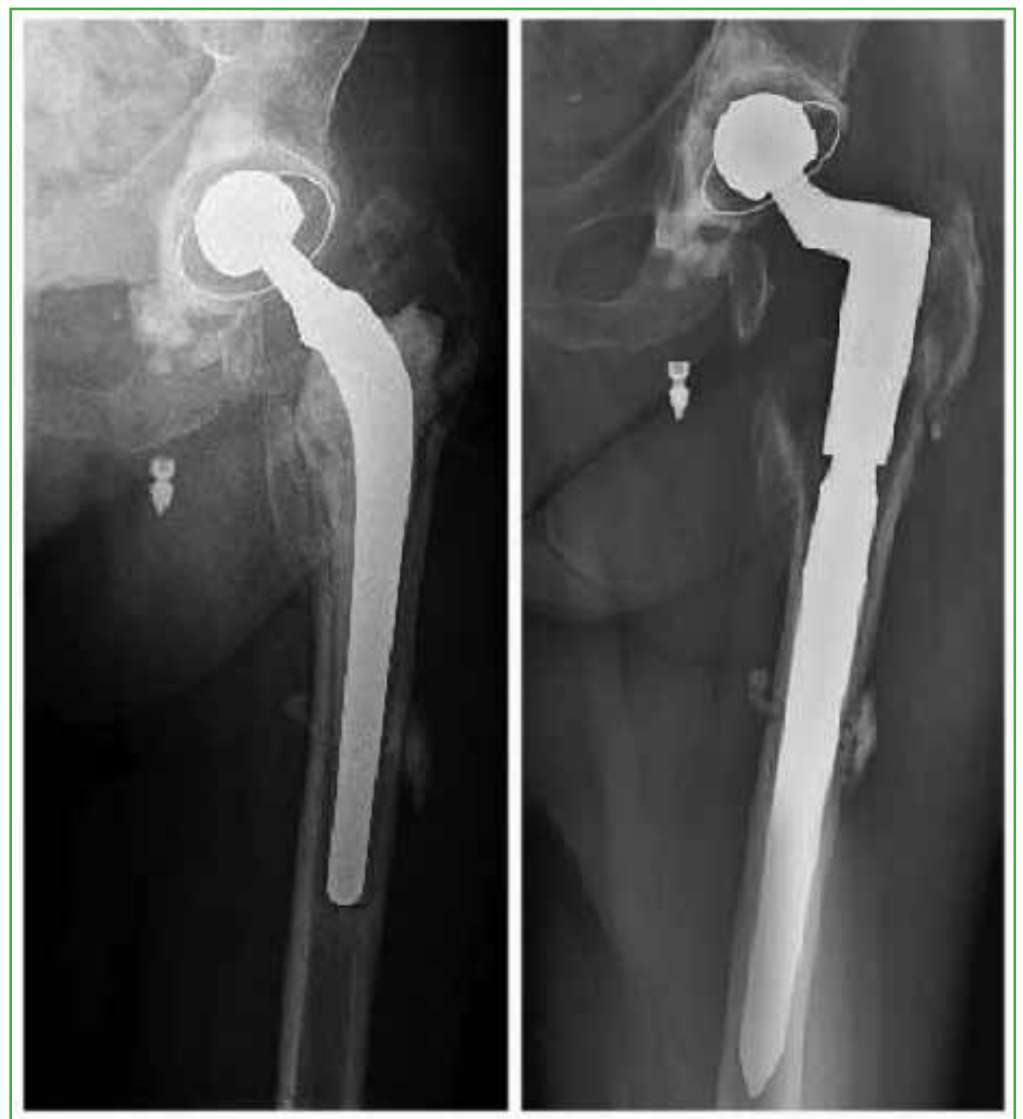

Figura 6. Mujer de 86 años. Aflojamiento aséptico del vástago femoral. Revisión femoral con vástago cónico estriado modular de fijación distal.

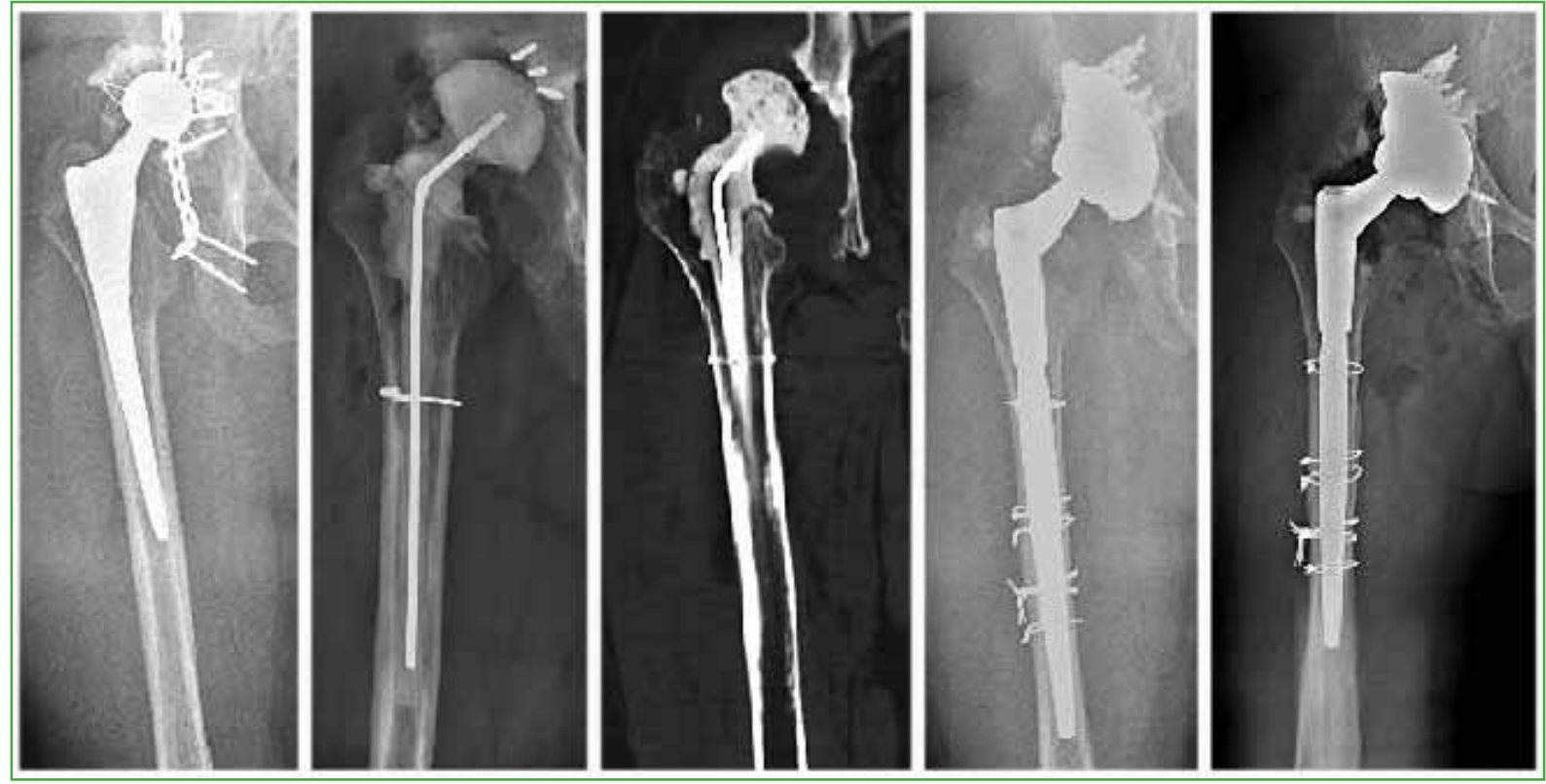

Figura 7. Hombre de 35 años. Secuela de fractura de acetábulo más necrosis ósea avascular. Aflojamiento séptico. Revisión con cotilo más aumento de metal trabecular y vástago cónico estriado modular de fijación distal. 


\section{DISCUSIÓN}

La revisión de una artroplastia total de cadera con déficit de stock óseo es un desafío para todo cirujano ortopedista. Los vástagos cónicos estriados modulares ofrecen varias ventajas, entre ellas, la estabilidad axial y rotatoria, así como la modularidad, la protección contra el stress shielding y una menor tasa de fracturas intraoperatorias y dolor en el muslo, comparados con los vástagos de cromo-cobalto con recubrimiento poroso extenso. ${ }^{16-17,19,20,30} \mathrm{El}$ principal desafío que enfrenta el cirujano ortopedista al efectuar este tipo de cirugías es lograr una fijación estable del componente femoral. Cuando se compromete el fémur proximal significativamente, ya sea por fractura o déficit de stock óseo, es necesaria la estabilización femoral distal a la zona de hueso debilitado para el éxito de la cirugía de revisión. ${ }^{23}$

En diferentes estudios, se ha comunicado el éxito de los vástagos cónicos estriados modulares. ${ }^{22,30}$ Nuestro estudio incluyó a 182 pacientes, y se trata de una de las series más grandes reportadas.

En la mayoría de los estudios publicados, se estima una supervivencia del implante sin revisión del componente femoral debido a aflojamiento aséptico del $98 \%$ a los 10 años. ${ }^{17,19}$ En nuestra cohorte, al final del seguimiento, el 95,05\% tenía un implante estable sin necesidad de una nueva cirugía, incluso en aquellos pacientes con déficits de stock óseo severos. Esto contrasta con la mayoría de los otros diseños de implantes femorales, como los vástagos no cementados con recubrimiento poroso extenso, cuyo éxito depende de la gravedad de la pérdida ósea. ${ }^{24}$

Abdel y cols..$^{31}$ comunicaron una tasa de subsidencia del 2,4\% de los vástagos implantados, y solo un paciente requirió reintervención quirúrgica. En nuestra serie, se observó esta complicación en el 11,5\% de los casos. Sin embargo, cuando analizamos el resto de la bibliografía, la tasa de subsidencia informada es similar a la de nuestra serie. ${ }^{19,28}$ Por el contrario, cuando se analizan los vástagos de cromo-cobalto con recubrimiento poroso extenso, estos valores son más altos. ${ }^{32}$

Klauser y cols. ${ }^{33}$ y Huang y cols. ${ }^{34}$ informaron una tasa de fracturas intraoperatorias de alrededor del $17 \%$. En nuestro estudio, solo el 2,19\% (4 pacientes) tuvo esta complicación.

En la mayoría de los estudios publicados, la tasa de inestabilidad secundaria a la revisión del componente femoral oscila entre el $2 \%$ y el $21 \% .^{18,19}$ En nuestra serie, fue del 7,6\%, lo que se correlaciona con los datos publicados.

Salcedo y cols. ${ }^{35}$ comunicaron una tasa de infección profunda del 2,5\%. Aunque la infección puede afectar negativamente la supervivencia del implante, esta complicación no es alta en la mayoría de las series publicadas. En nuestro estudio, solo cuatro pacientes necesitaron una reintervención por esta complicación posquirúrgica.

Una limitación de nuestro estudio es que se trata de una serie retrospectiva de casos, no aleatorizado y sin un grupo de control.

\section{CONCLUSIONES}

Los vástagos cónicos estriados modulares de fijación distal proporcionan una solución reproducible para el manejo de revisiones de componentes femorales. En nuestra serie, esta técnica de revisión femoral logró una alta tasa de éxito con buenos resultados funcionales. La tasa de complicaciones fue similar a la reportada en la literatura. Es importante destacar que la planificación preoperatoria y la selección adecuada del diámetro del vástago son esenciales para evitar complicaciones. Sin embargo, es necesario realizar un seguimiento más extenso de los pacientes para asegurarse de que las complicaciones asociadas con la modularidad, como la corrosión, no sean un problema a futuro.

Conflicto de intereses: Los autores no declaran conflictos de intereses.

ORCID de F. Vanoli: https://orcid.org/0000-0002-5667-9716

ORCID de L. Pérez Alamino: https://orcid.org/0000-0002-1563-6947

ORCID de I. Pioli: https://orcid.org/0000-0001-8697-1980

ORCID de J. Gómez: https://orcid.org/0000-0002-1162-2708
ORCID de F. A. Lopreite: https://orcid.org/0000-0002-2065-8649

ORCID de P. Maletti: https://orcid.org/0000-0001-5065-9880

ORCID de H. del Sel: https://orcid.org/0000-0002-3655-1408

ORCID de B. L. Allende: https://orcid.org/0000-0003-2757-4381 


\section{BIBLIOGRAFÍA}

1. Kurtz S, Ong K, Lau E, Mowat F, Halpern M. Projections of primary and revision hip and knee arthroplasty in the United States from 2005 to 2030. J Bone Joint Surg Am 2007;89(4):780-5. https://doi.org/10.2106/JBJS.F.00222

2. Gie GA, Linder L, Ling RS, Simon JP, Slooff TJ, Timperley AJ. Impacted cancellous allografts and cement for revision total hip arthroplasty. J Bone Joint Surg Br 1993;75(1):14-21. https://doi.org/10.1302/0301-620X.75B1.8421012

3. Engh CA, Glassman AH, Griffin WL, Mayer JG. Results of cementless revision for failed cemented total hip arthroplasty. Clin Orthop Relat Res 1988;(235):91-110. PMID: 3416544

4. Krishnamurthy AB, MacDonald SJ, Paprosky WG. 5- to 13-year follow-up study on cementless femoral components in revision surgery. J Arthroplasty 1997;12(8):839-47. https://doi.org/10.1016/s0883-5403(97)90152-2

5. Lawrence JM, Engh CA, Macalino GE, Lauro GR. Outcome of revision hip arthroplasty done without cement. $J$ Bone Joint Surg 1994;76(7):965-73. https://doi.org/10.2106/00004623-199407000-00002

6. Wimmer MD, Randau TM, Deml MC, Ascherl R, Nöth U, Forst R, et al. Impaction grafting in the femur in cementless modular revision total hip arthroplasty: a descriptive outcome analysis of 243 cases with the MRPTITAN revision implant. BMC Musculoskelet Disord 2013;14(1):19. https://doi.org/10.1186/1471-2474-14-19

7. Oshima S, Yasunaga Y, Yamasaki T, Yoshida T, Hori J, Ochi M. Midterm results of femoral impaction bone grafting with an allograft combined with hydroxyapatite in revision total hip arthroplasty. J Arthroplasty 2012;27(3):470-6. https://doi.org/10.1016/j.arth.2011.07.012

8. Ten Have BLEF, Brouwer MD RW, van Biezen FC, Verhaar JAN. Femoral revision surgery with impaction bone grafting: 31 hips followed prospectively for ten to 15 years. J Bone Joint Surg Br 2012;94(5):615-8. https://doi.org/10.1302/0301-620X.94B5.27909

9. Mulroy WF, Harris WH. Revision total hip arthroplasty with use of so-called second-generation cementing techniques for aseptic loosening of the femoral component. A fifteen-year-average follow-up study. J Bone Joint Surg 1996;78(3):325-30. https://doi.org/10.2106/00004623-199603000-00002

10. Davis CM, Berry DJ, Harmsen WS. Cemented revision of failed uncemented femoral components of total hip arthroplasty. J Bone Joint Surg Am 2003;85(7):1264-9. https://doi.org/10.2106/00004623-200307000-00012

11. Lin Min, Jing Peng, Hong Duan, Wenli Zhang, Yong Zhou, Chongqi Tu. Uncemented allograft-prosthetic composite reconstruction of the proximal femur. Indian J Orthop 2014;48(3):289-95. https://doi.org/10.4103/0019-5413.132521

12. Mayle Jr RE, Paprosky WG. Massive bone loss: Allograft-prosthetic composites and beyond. J Bone Joint Surg Br 2012;94(11 Suppl A):61-4. https://doi.org/10.1302/0301-620X.94B11.30791

13. Kato T, Otani T, Sugiyama H, Hayama T, Katsumata S, Marumo K. Cementless total hip arthroplasty in hip dysplasia with an extensively porous-coated cylindrical stem modified for Asians: A 12-year follow-up study. $J$ Arthroplasty 2015;30(6):1014-8. https://doi.org/10.1016/j.arth.2015.01.033

14. Shen B, Huang Q, Yang J, Zhou Z, Kang P, Pei F. Extensively coated non-modular stem used in two-stage revision for infected total hip arthroplasty: Mid-term to long-term follow-up: non-modular stem for infected THA. Orthop Surg 2014;6(2):103-9. https://doi.org/10.1111/os.12107

15. Thomsen PB, Jensen NJF, Kampmann J, Hansen TB. Revision hip arthroplasty with an extensively porous-coated stem - Excellent long-term results also in severe femoral bone stock loss. Hip Int 2013;23(4):352-8. https://doi.org/10.5301/hipint.5000032

16. Abdel MP, Lewallen DG, Berry DJ. Periprosthetic femur fractures treated with modular fluted, tapered stems. Clin Orthop Relat Res 2014;472(2):599-603. https://doi.org/10.1007/s11999-013-2936-4

17. Van Houwelingen AP, Duncan CP, Masri BA, Greidanus NV, Garbuz DS. High survival of modular tapered stems for proximal femoral bone defects at 5 to 10 years followup. Clin Orthop Relat Res 2013;471(2):454-62. https://doi.org/10.1007/s11999-012-2552-8

18. Amanatullah DF, Howard JL, Siman H, Trousdale RT, Mabry TM, Berry DJ. Revision total hip arthroplasty in patients with extensive proximal femoral bone loss using a fluted tapered modular femoral component. Bone Joint $J$ Br 2015;97(3):312-7. https://doi.org/10.1302/0301-620X.97B3.34684

19. Rodriguez JA, Deshmukh AJ, Robinson J, Cornell CN, Rasquinha VJ, Ranawat AS, et al. Reproducible fixation with a tapered, fluted, modular, titanium stem in revision hip arthroplasty at 8-15 years follow-up. J Arthroplasty 2014;29(9):214-8. https://doi.org/10.1016/j.arth.2013.12.035

20. Munro JT, Garbuz DS, Masri BA, Duncan CP. Tapered fluted titanium stems in the management of Vancouver B2 and B3 periprosthetic femoral fractures. Clin Orthop Relat Res 2014;472(2):590-8.

https://doi.org/10.1007/s11999-013-3087-3 
21. Abdel MP. CORR Insights®: Can a conical implant successfully address complex anatomy in primary THA? Radiographs and hip scores at early followup. Clin Orthop Relat Res 2016;474(2):465-6. https://doi.org/10.1007/s11999-015-4530-4

22. Park M-S, Lee J-H, Park J-H, Ham D-H, Rhee Y-K. A distal fluted, proximal modular femoral prosthesis in revision hip arthroplasty. J Arthroplasty 2010;25(6):932-8. https://doi.org/10.1016/j.arth.2009.06.031

23. Sculco PK, Abdel MP, Hanssen AD, Lewallen DG. The management of bone loss in revision total knee arthroplasty: rebuild, reinforce, and augment. Bone Joint J 2016;98-B(1 Suppl A):120-4. https://doi.org/10.1302/0301-620X.98B1.36345

24. Paprosky WG, Burnett RSJ. Assessment and classification of bone stock deficiency in revision total hip arthroplasty. Am J Orthop (Belle Mead NJ) 2002;31(8):459-64. PMID: 12216969

25. Ling RS, Timperley AJ, Linder L. Histology of cancellous impaction grafting in the femur. A case report. J Bone Joint Surg Br 1993;75(5):693-6. https://doi.org/10.1302/0301-620X.75B5.8376422

26. Duncan CP, Masri BA. Fractures of the femur after hip replacement. Instr Course Lect 1995;44:293-304. PMID: 7797866

27. Callaghan JJ, Salvati EA, Pellicci PM, Wilson PD Jr, Ranawat CS. Results of revision for mechanical failure after cemented total hip replacement. 1979 to 1982. A two to five-year follow-up. J Bone Joint Surg Am 1985;67:107485. PMID: 4030826

28. Park M-S, Lim Y-J, Chung W-C, Ham D-H, Lee S-H. Management of periprosthetic femur fractures treated with distal fixation using a modular femoral stem using an anterolateral approach. J Arthroplasty 2009;24(8):1270-6. https://doi.org/10.1016/j.arth.2009.07.013

29. Harris WH. Traumatic arthritis of the hip after dislocation and acetabular fractures: treatment by mold arthroplasty. An end-result study using a new method of result evaluation. J Bone Joint Surg Am 1969;51(4):737-55. PMID: 5783851

30. Fink B, Urbansky K, Schuster P. Mid term results with the curved modular tapered, fluted titanium Revitan stem in revision hip replacement. Bone Joint J 2014;96-B(7):889-95. https://doi.org/10.1302/0301-620X.96B7.33280

31. Abdel MP, Cottino U, Larson DR, Hanssen AD, Lewallen DG, Berry DJ. Modular fluted tapered stems in aseptic revision total hip arthroplasty. J Bone Joint Surg 2017;99(10):873-81. https://doi.org/10.2106/JBJS.16.00423

32. Garcia-Cimbrelo E, Garcia-Rey E, Cruz-Pardos A, Madero R. Stress-shielding of the proximal femur using an extensively porous-coated femoral component without allograft in revision surgery: a 5- to 17-year follow-up study. J Bone Joint Surg Br 2010;92(10):1363-9. https://doi.org/10.1302/0301-620X.92B10.24317

33. Klauser W, Bangert Y, Lubinus P, Kendoff D. Medium-term follow-up of a modular tapered noncemented titanium stem in revision total hip arthroplasty: a single-surgeon experience. J Arthroplasty 2013;28(1):84-9. https://doi.org/10.1016/j.arth.2012.07.001

34. Huang Y, Shao H, Zhou Y, Gu J, Tang H, Yang D. Femoral bone remodeling in revision total hip arthroplasty with use of modular compared with monoblock tapered fluted titanium stems: the role of stem length and stiffness. $J$ Bone Joint Surg Am 2019;101(6):531-8. https://doi.org/10.2106/JBJS.18.00442

35. Salcedo Zunino LA, Núñez J, Mangupli MM, Pioli I, Gomez J, Allende BL. Revisión femoral con tallo cónico de fijación distal. Rev Asoc Argent Ortop Traumatol 2017;82(1):40-2. https://www.aaot.org.ar/revista/2017/n1/7.pdf 